\title{
Small Fermi Surface Pockets in Underdoped High Temperature Superconductors: Observation of Shubnikov-de Haas Oscillations in $\mathrm{YBa}_{2} \mathrm{Cu}_{4} \mathrm{O}_{8}$
}

\author{
A. F. Bangura, ${ }^{1}$ J. D. Fletcher, ${ }^{1}$ A. Carrington, ${ }^{1}$ J. Levallois, ${ }^{2}$ M. Nardone,${ }^{2}$ B. Vignolle, ${ }^{2}$ P. J. Heard, ${ }^{1}$ N. Doiron-Leyraud, ${ }^{3}$ \\ D. LeBoeuf, ${ }^{3}$ L. Taillefer, ${ }^{3}$ S. Adachi, ${ }^{4}$ C. Proust, ${ }^{2}$ and N. E. Hussey ${ }^{1}$ \\ ${ }^{1}$ H. H. Wills Physics Laboratory, University of Bristol, Tyndall Avenue, BS8 1TL, United Kingdom \\ ${ }^{2}$ Laboratoire National des Champs Magnétiques Pulsés, UMR CNRS-UPS-INSA 5147, Toulouse, France \\ ${ }^{3}$ Département de physique and RQMP, Université de Sherbrooke, Sherbrooke, JIK 2RI, Canada \\ ${ }^{4}$ Superconducting Research Laboratory, International Superconductivity Center, Shinonome 1-10-13, Tokyo 135, Japan
}

(Received 30 July 2007; published 1 February 2008)

We report the observation of Shubnikov-de Haas oscillations in the underdoped cuprate superconductor $\mathrm{YBa}_{2} \mathrm{Cu}_{4} \mathrm{O}_{8}$ (Y124). For fields aligned along the $c$ axis, the frequency of the oscillations is $660 \pm$ $30 \mathrm{~T}$, which corresponds to $\sim 2.4 \%$ of the total area of the first Brillouin zone. The effective mass of the quasiparticles on this orbit is measured to be $2.7 \pm 0.3$ times the free electron mass. Both the frequency and mass are comparable to those recently observed for ortho-II $\mathrm{YBa}_{2} \mathrm{Cu}_{3} \mathrm{O}_{6.5}$ (Y123-II). We show that although small Fermi surface pockets may be expected from band-structure calculations in Y123-II, no such pockets are predicted for Y124. Our results therefore imply that these small pockets are a generic feature of the copper oxide plane in underdoped cuprates.

DOI: 10.1103/PhysRevLett.100.047004

Understanding the doping evolution of the electronic ground state within the superconducting $\mathrm{CuO}_{2}$ planes is central to the high- $T_{c}$ problem, yet many outstanding issues remain. Heavily overdoped cuprates, for example, behave in many ways like conventional, highly anisotropic metals [1-3], yet quantum oscillations, a characteristic signature of a Fermi-liquid ground state [4], have never been observed. Moreover, it is usually assumed that as hole doping is decreased and the materials evolve towards the antiferromagnetic insulating state, the properties depart substantially from those of a conventional Fermi-liquid. The recent observation [5] of the Shubnikov-de Haas effect $(\mathrm{SdH})$ in underdoped $\mathrm{YBa}_{2} \mathrm{Cu}_{3} \mathrm{O}_{6.5}$, with $T_{c} / T_{c}^{\max } \simeq$ 0.6 , was therefore a surprising and highly significant discovery.

The frequency of the $\mathrm{SdH}$ oscillations in oxygen ordered ortho-II $\mathrm{YBa}_{2} \mathrm{Cu}_{3} \mathrm{O}_{6.5}$ (Y123-II) corresponds to a Fermi surface pocket with a cross-sectional area (for $\mathbf{H} \| c$ ) that is only a small fraction $(\sim 2 \%)$ of the total Brillouin zone area. The angle dependence of the frequency of this oscillation suggests that this pocket is quasi-two-dimensional [6]. Density functional theory (DFT) band-structure calculations $[7,8]$ suggest that these pockets may be formed from a flat band, originating from the $\mathrm{CuO}$ chain and $\mathrm{BaO}$ layers, which lies very close to the Fermi level $E_{F}$. However, there are a number of alternative possibilities such as the formation of pockets near the nodal points of the DFT $\mathrm{CuO}_{2}$ plane Fermi surface, where the $d$-wave superconducting gap is minimal, and where the most intense spectral weight is observed by angle resolved photoemission spectroscopy (ARPES) [9]. These pockets or "Fermi arcs" may occur due to promixity to the Mott insulating state [10] or because of reconstruction of the original Fermi surface resulting from the formation of an ordered phase with broken symmetry [11-13]. Clearly, it is
PACS numbers: 74.25.Jb, 71.18.+y, 74.25.Ha, 74.72.Bk

imperative to determine whether the $\mathrm{SdH}$ signals are unique to Y123-II or are an intrinsic feature of the electronic structure of the cuprates.

In this Letter we report the observation of SdH oscillations in another underdoped cuprate superconductor $\mathrm{YBa}_{2} \mathrm{Cu}_{4} \mathrm{O}_{8}(\mathrm{Y} 124)$, which has a higher $T_{c}(\sim 80 \mathrm{~K})$ and a higher hole density per planar $\mathrm{Cu}(p \sim 0.14)$ than Y123II $(p \sim 0.1)$, in pulsed high magnetic fields up to $61 \mathrm{~T}$. The frequency of the oscillations is comparable with those in Y123-II. This similarity, coupled with the marked differences in the calculated band structure of the two materials, strongly points to an origin of the oscillations which is generic to the underdoped cuprates.

In contrast to the $\mathrm{Y} 123$ family, which has a single $\mathrm{CuO}$ chain with variable oxygen content, Y124 contains alternating stacks of $\mathrm{CuO}_{2}$ bilayers and double $\mathrm{CuO}$ chains that are stable and fully loaded. Single crystalline samples (typical dimensions $400 \times 80 \times 30 \mu \mathrm{m}^{3}$ ) were flux grown in $\mathrm{Y}_{2} \mathrm{O}_{3}$ crucibles in a partial oxygen pressure of $400 \mathrm{bar}$ [14]. Magnetoresistance (MR) measurements were performed in the LNCMP pulsed field facility in Toulouse. Although several samples were measured, clear SdH signals were seen in only two samples with the highest $T_{c}$ values $(>80 \mathrm{~K})$ and the largest MR response. Four-point resistance measurements were taken at a frequency of 40$80 \mathrm{kHz}$ with the current (10-20 mA) applied I $\| a$ (sample No. $1, T_{c} \sim 81 \mathrm{~K}$ ) and $\mathbf{I} \| b$ (sample No. $2, T_{c} \sim 82 \mathrm{~K}$ ). The contact geometry for each crystal is shown in the insets of Fig. 1. The voltage across the samples was recorded using an analogue to digital converter allowing the data to be postprocessed. The samples were cooled with either a dilution fridge (No. 1) or a pumped ${ }^{4} \mathrm{He}$ cryostat (No. 2) housed in two different magnet coils. In both cases, the samples were in direct contact with the cryogenic liquid at all temperatures. The magnet coils had peak fields of 58 


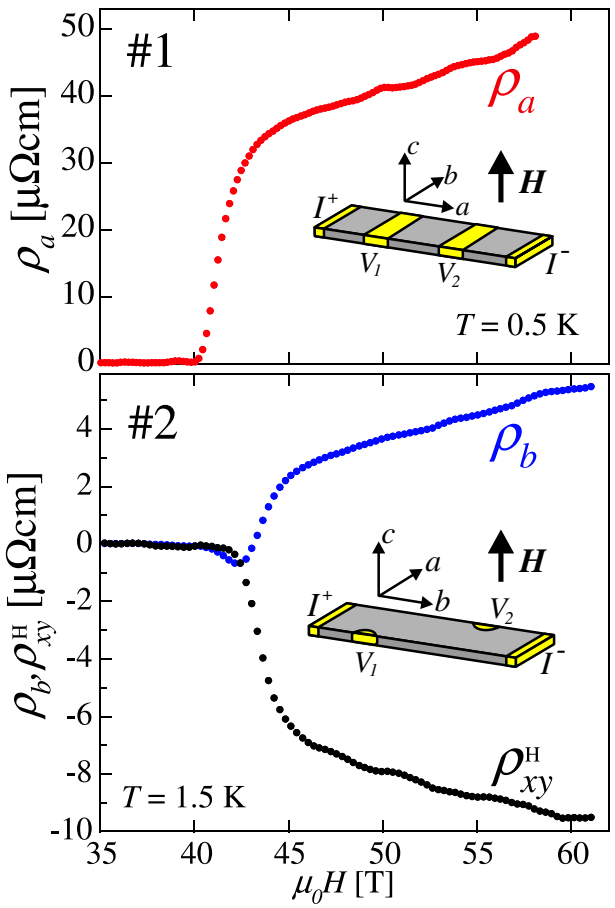

FIG. 1 (color online). Longitudinal and Hall data $\left(\rho_{x x}, \rho_{y y}\right.$ and $\rho_{x y}$ ) for two Y124 samples. The insets show the contact configuration for each sample.

and $61 \mathrm{~T}$, respectively, and in the field range of interest here (45-61 T), the maximum field rise or fall rates were $\sim 2000 \mathrm{~T} / \mathrm{s}$. Data for up and down sweeps showed negligible hysteresis.

The contact configuration of sample No. $2(\mathbf{I} \| b)$ allowed us to measure both the longitudinal $\left(\rho_{y y}\right)$ and Hall $\left(\rho_{x y}^{H}\right)$ resistivities by reversing the direction of the applied field, whereas for sample No. 1 (I $\| a), \rho_{x x}$ was measured directly. In order to improve the signal to noise ratio, data from several (typically 2-3) pulses were averaged. Resistivity versus field data for both samples are shown in Fig. 1. The resistivities rise rapidly above the irreversibility field $H_{\text {irr }}$ (which is slightly different in the two samples) then increase more slowly. Above $H_{\text {irr }}$, the resistivity is highly anisotropic within the plane due to the presence of the highly conducting double-chain unit, as found in zero-field above $T_{c}$ [15]. As in Y123-II [5], $\rho_{x y}^{H}$ is negative and varies linearly with $H$, extrapolating to the origin. This linear field dependence suggests that the negative $\rho_{x y}$ is a normal state effect, perhaps resulting from a Fermi surface reconstruction [16] as discussed below, rather than a mixed state effect. For both samples and in both the longitudinal and transverse components, small period (in inverse field) $\mathrm{SdH}$ oscillations are visible which are a few percent of the total signal at maximum field.

In Fig. 2 we show the oscillatory component of the MR signal for both crystals at various temperatures. Because of small differences in noise level for the two field directions, our study of the $T$ dependence of the oscillations in sample

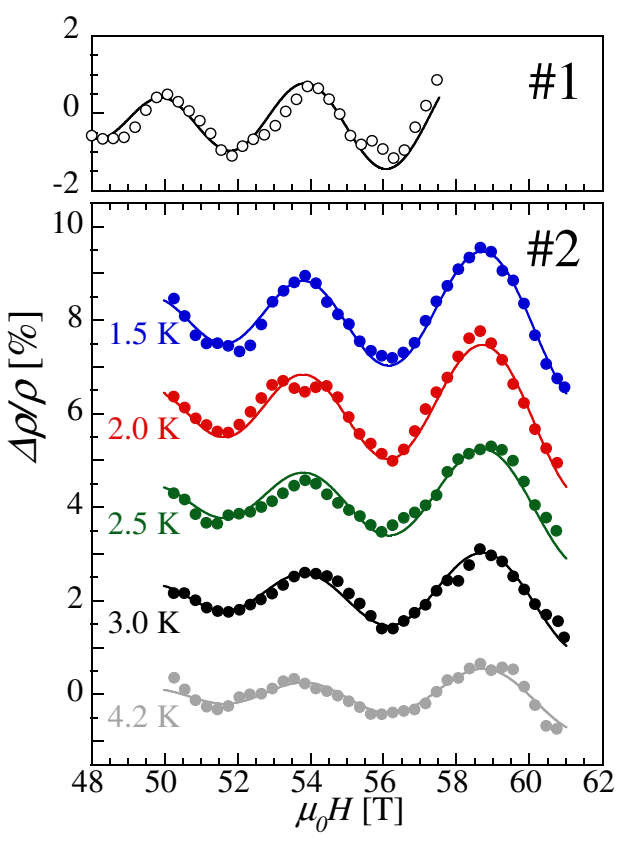

FIG. 2 (color online). The oscillatory component of the magnetoresistance $[\Delta \rho / \rho(H)]$ for both samples at various temperatures. For No. $1, \rho=\rho_{x x}(H)$ while for No. 2, $\rho$ is a mixture of $\rho_{y y}(H)$ and $\rho_{x y}^{H}(H)$. In each case, a linear background has been subtracted and the data offset for clarity. The solid lines are fits to Eq. (1) giving $m^{*}=2.7 \pm 0.3 m_{e}$.

No. 2 was carried out with the field in one direction only. The resistivity $\rho$ shown in Fig. 2 for No. 2 is therefore a mixture of $\rho_{y y}$ and $\rho_{x y}^{H}$.

We analyzed the data using the standard theory for $\mathrm{SdH}$ oscillations in a three-dimensional metal [17], which is essentially the same as the Lifshitz-Kosevich model for the de Haas-van Alphen oscillations in the magnetization [4]

$$
\frac{\Delta \rho}{\rho}=A H^{1 / 2} R_{T} R_{D} \sin \left(\frac{2 \pi F}{B}+\phi\right),
$$

where $A$ is a constant. The temperature damping factor $R_{T}=X /(\sinh X)$ with $X=\left(2 \pi^{2} k_{B} / \hbar e\right)\left(m^{*} T / B\right)$ and $m^{*}$ is the quasiparticle effective mass. The Dingle factor $R_{D}=$ $\exp \left(-\pi / \omega_{c} \tau\right)=\exp \left[-\left(\pi \hbar k_{F}\right) /(e B \ell)\right]$ describes the increase in damping as the orbitally averaged mean-freepath $\ell$ decreases or as the average Fermi wave vector $\left(k_{F}\right)$ of the orbit grows.

A fit of this equation to the data in Fig. 2 gives a single $\mathrm{SdH}$ frequency $F$ of $660 \pm 30 \mathrm{~T}$ for both samples. Using the Onsager relation, $F=(\hbar / 2 \pi e) \mathcal{A}$, we find that $\mathcal{A}$, the cross-sectional area of this orbit, corresponds to $2.4 \%$ of the average cross-sectional area of the first Brillouin zone (27.9 kT for $\mathbf{H} \| c$ ). Assuming that the Fermi surface or pocket corresponding to this orbit is two-dimensional, and that there are $n$ such pockets per $\mathrm{CuO}_{2}$ sheet, Luttinger's theorem gives $p=n a b \mathcal{A} / 4 \pi^{2}=0.024$ (2) $n$ (where $a, b$ are the lattice constants). More importantly, $\mathcal{A}$ is found to be $1.25 \pm 0.07$ larger than that observed in Y123-II for which $p \sim 0.10$. Although it is difficult to pinpoint pre- 
cisely the actual number of doped holes $p$ per $\mathrm{CuO}_{2}$ plane in Y124, taking the average of $p$ from three independent estimates (by comparing $T_{c}$ [18] and the pseudogap temperature $T^{*}[15,19,20]$ with Y123 and the Seebeck coefficient [21,22]), one obtains $p \sim 0.14$. Thus $\mathcal{A}$ is found to scale approximately linearly with the nominal hole densities of Y123-II and Y124.

As a first approach for determining $m^{*}$, we neglect the field dependence of $R_{T}$ and fix $\omega_{c} \tau / B, F$ and $\phi$ to the values found at the lowest temperature so that changes in the values of $A$ represent $R_{T}$ at an effective field $B_{e}=$ $55 \mathrm{~T}$. A fit of $R_{T}$ to $A(T)$ is shown in Fig. 3 and gives $m^{*}=$ $2.5 \pm 0.3 m_{e}$, where $m_{e}$ is the free electron mass. This approach slightly underestimates the mass, and a better approach is to fit all the data at all temperatures to Eq. (1) simultaneously. These fits, shown by solid lines in Fig. 2, give $m^{*}=2.7 \pm 0.3 m_{e}$. This is a heavier mass than that observed in Y123-II by a factor $1.4 \pm 0.2$.

Fast Fourier transforms of $\rho(1 / B, T)$ are shown in the inset to Fig. 3. Both the frequency of the main peak and the mass derived from the $T$-dependent amplitude are consistent with the direct fitting described above, although the former technique is more accurate when so few oscillations are present.

From our fits in Fig. 2, we obtain a Dingle factor $\left(e^{-\alpha / B}\right.$, $\alpha=340 \pm 100 \mathrm{~T}^{-1}$ ) for sample No. 2 which for cylindrical pockets gives an average $\mathrm{SdH}$ mean-free-path $\ell_{\mathrm{SdH}} \simeq$ $90 \pm 30 \AA$ A. Unfortunately, we cannot obtain an independent estimate of $\ell_{\text {tr }}$, the transport mean-free path of the inplane carriers, as we do not yet know how many pockets are present. For sample No. 1, the magnitude of the zerofield residual in-plane resistivity $\rho_{a 0} \simeq 6 \pm 2 \mu \Omega \mathrm{cm}$, estimated by fitting the high-field data to the expression $\rho_{a}(H)=\rho_{a 0}+A H^{2} /\left(1+B H^{2}\right)$ that describes the form

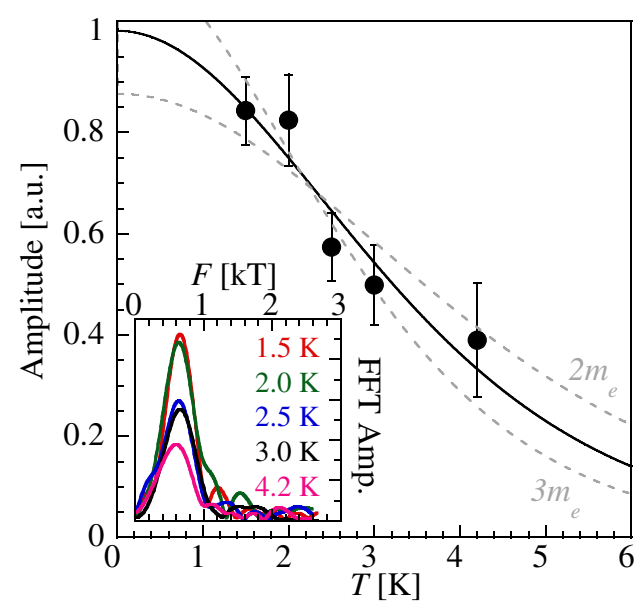

FIG. 3 (color online). SdH signal amplitude versus temperature for sample No. 2. The solid line is a fit to the standard expression for the temperature damping factor, $R_{T}$, yielding an effective mass of $2.5 \pm 0.3 m_{e}$. The faint dashed lines give the approximate range of masses consistent with the present data. Inset: Fast Fourier transform of $\rho(1 / B, T)$. of the MR beyond the weak-field limit [23]. Assuming there are $n$ pockets per $\mathrm{CuO}_{2}$ plane (and $2 n$ in total), this would give $\ell_{\mathrm{tr}}=h c / 2 n k_{F} e^{2} \rho_{a 0}=2000 / n \AA \quad( \pm 30 \%)$. Here, $c=13.6 \AA$ is the $c$ axis lattice spacing and $k_{F}=$ $1.4 \mathrm{~nm}^{-1}$, as determined from the $\mathrm{SdH}$ frequency.

The above analysis implies that there is either a large number of Fermi pockets in Y124, or that the Dingle factor grossly underestimates the transport lifetime [24]. The latter could be attributed either to the stronger effect of small-angle scattering on the dephasing of quantum oscillations, as seen in simple metals [17], or to additional damping in the superconducting mixed state. Quantum oscillations have been observed in the superconducting state of a number of different materials (see, for example, Refs. [25,26]). Whilst the frequencies and effective masses are found to be the same as in the normal state, an additional damping is observed which is related to the magnitude of the field dependent energy gap. We note however, that if the orbits observed here originated from the nodal regions of the Fermi surface, as suggested by ARPES [9], then it may be expected that this additional damping is small because of the $d$-wave symmetry of the superconducting gap function.

As mentioned above, conventional DFT band-structure calculations $[7,8]$ for Y123-II show that with small rigid shifts of bands ( $\Delta E \sim \pm 20 \mathrm{meV}$ ), a flat band arising from the $\mathrm{CuO}$ chain layer and apical oxygen ( $\mathrm{BaO}$ layer) can break through $E_{F}$ giving rise to small pockets, with frequencies rising up to $\sim 500 \mathrm{~T}$ and bare masses $\sim 1-3 m_{e}$. To investigate the possibility of similar pockets arising in Y124 we calculated the band-structure of Y124 using the WIEN2K package [27], which is an implementation of a fullpotential, augmented plane wave plus local orbital scheme. The crystal structure from Ref. [28], and $10^{4} k$ points in the full Brillouin zone were used for the consistency cycle.

The band structure and Fermi surface are shown in Fig. 4 and are essentially the same as those reported previously $[29,30]$. The Fermi surface consists of two large holelike quasi-2D cylinders centered on the zone corner $(J)$ with mostly plane character and two warped quasi-1D sheets with mostly chain character. Crucially, the position of the above mentioned $\mathrm{CuO} / \mathrm{BaO}$ band is now $\sim 400 \mathrm{meV}$ below $E_{F}$ (at the $J$ point), and so a very large shift of the energy of this band would be needed to produce Fermi surface pockets of similar origin to those calculated for Y123-II. In fact, $E_{F}$ needs to be shifted down by $\sim 80 \mathrm{meV}$ before any new closed orbits are formed for $\mathbf{H} \| c$. The orbit formed is centered around the zone center $(\Gamma)$ [see Fig. 4(d)] and has a high frequency $\left(\sim 3500 \mathrm{~T}\right.$ for $\Delta E_{F}=$ $-100 \mathrm{meV}$ ) so cannot feasibly be responsible for our observations. Given the similarity of our $\mathrm{SdH}$ results for Y124 and those for Y123-II, it therefore seems unlikely that the oscillations in either material can be explained by conventional band-structure theory.

As proposed for Y123-II [5], an alternative to the conventional band-structure approach is to associate our $\mathrm{SdH}$ 


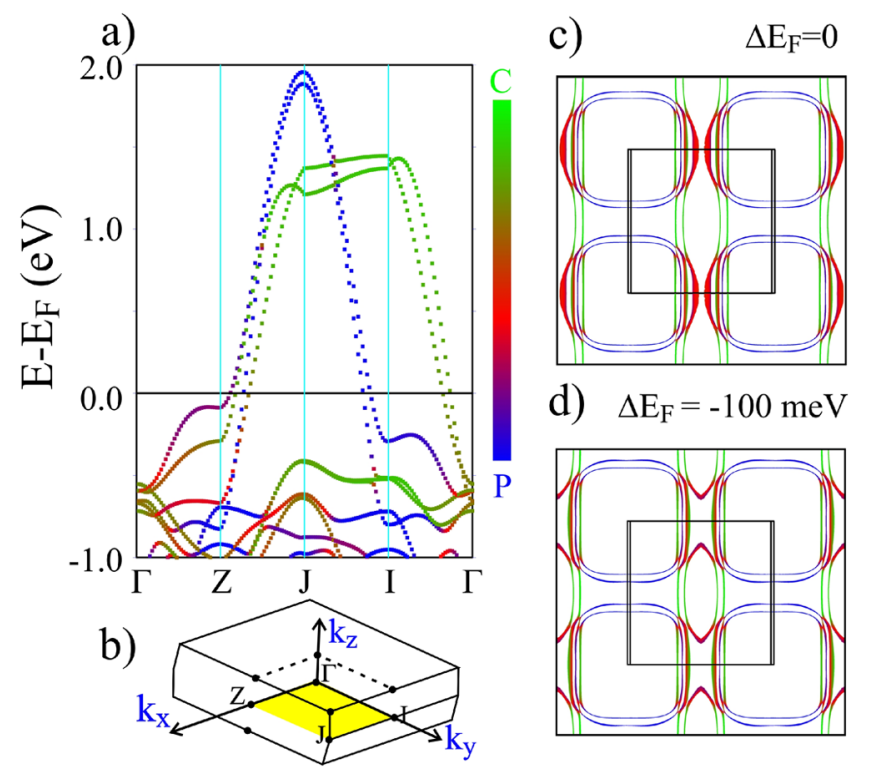

FIG. 4 (color online). (a) Band-structure of Y124 along symmetry directions in the basal plane. The color scale reflects the band character: $C=\mathrm{CuO}$ chain character (including apical oxygen) $P=\mathrm{CuO}_{2}$ plane character. (b) Brillouin zone of the Ammm space group of Y124, showing the symmetry points labeled in a). (c) Fermi surface of Y124 viewed along the $c$ axis. The shaded region is the first Brillouin zone. (d) As for (c) but with $E_{F}$ shifted down by $100 \mathrm{meV}$.

orbits with Fermi surface reconstruction, due, e.g., to spindensity-wave [11,13] or $d$-density-wave [12] formation. Unfortunately, it is not possible at this stage to have a complete picture of the (reconstructed) Fermi surface until we know precisely how many $\mathrm{SdH}$ orbits are present in Y124. In this regard, measurements to higher fields should prove very informative.

In summary, we have observed Shubnikov-de Haas oscillations in the underdoped cuprate Y124. The frequency of the oscillations is comparable with those observed in Y123-II, scaling approximately by the ratio of hole doping levels in the two compounds. It is likely that these oscillations are a generic feature of underdoped cuprates perhaps resulting from a reconstruction of the $\mathrm{CuO}_{2}$ plane Fermi surface, although as both materials in which SdH oscillations have been observed so far (Y123-II and Y124) contain quasi-1D $\mathrm{CuO}$ chain structures, an alternative origin cannot be completely ruled out at this time. Nevertheless our results provide precise information on how the pocket area and effective masses vary as a function of doping, providing a strong constraint on theories which seek to explain their electronic structure.

We thank E. A. Yelland and J. R. Cooper for showing us their data prior to publication [31], and G. G. Lonzarich, J. W. Loram, and S. M. Hayden for useful discussions. We also thank EAY for help with the band-structure calculations. This work was supported by the EPSRC (U.K.), NSERC (Canada), CIAR (Canada) and by Euromagnet (EU Contract No. 506239).
[1] C. Proust, E. Boaknin, R. W. Hill, L. Taillefer, and A. P. Mackenzie, Phys. Rev. Lett. 89, 147003 (2002).

[2] S. Nakamae et al., Phys. Rev. B 68, 100502(R) (2003).

[3] N. E. Hussey et al., Nature (London) 425, 814 (2003).

[4] D. Shoenberg, Magnetic Oscillations in Metals (Cambridge University Press, Cambridge, 1984).

[5] N. Doiron-Leyraud et al., Nature (London) 447, 565 (2007).

[6] C. Jaudet et al., arXiv:0711.3559.

[7] A. Carrington and E. A. Yelland, Phys. Rev. B 76, 140508(R) (2007).

[8] I.S. Elfimov, G. A. Sawatzky, and A. Damascelli, arXiv:0706.4276.

[9] A. Damascelli, Z. Hussain, and Z.X. Shen, Rev. Mod. Phys. 75, 473 (2003).

[10] B. Kyung et al., Phys. Rev. B 73, 165114 (2006).

[11] J. Lin and A. J. Millis, Phys. Rev. B 72, 214506 (2005).

[12] S. Chakravarty, R. B. Laughlin, D. K. Morr, and C. Nayak, Phys. Rev. B 63, 094503 (2001).

[13] W.Q. Chen, K. Y. Yang, T. M. Rice, and F. C. Zhang, arXiv:0706.3556.

[14] S. Adachi et al., Physica (Amsterdam) 301C, 123 (1998).

[15] N.E. Hussey, K. Nozawa, H. Takagi, S. Adachi, and K. Tanabe, Phys. Rev. B 56, R11 423 (1997).

[16] D. LeBoeuf et al., Nature (London) 450, 533 (2007).

[17] F. E. Richards, Phys. Rev. B 8, 2552 (1973).

[18] R. X. Liang, D. A. Bonn, and W. N. Hardy, Phys. Rev. B 73, 180505(R) (2006).

[19] K. Takenaka, K. Mizuhashi, H. Takagi, and S. Uchida, Phys. Rev. B 50, 6534 (1994).

[20] K. Segawa and Y. Ando, Phys. Rev. Lett. 86, 4907 (2001).

[21] J. L. Tallon, C. Bernhard, H. Shaked, R. L. Hitterman, and J. D. Jorgensen, Phys. Rev. B 51, 12911 (1995).

[22] J.S. Zhou, J. B. Goodenough, and B. Dabrowski, Phys. Rev. B 58, R2956 (1998).

[23] A. W. Tyler et al., Phys. Rev. B 57, R728 (1998).

[24] We note that for carriers within the chains, $\ell_{\text {tr }}$ can be estimated indirectly from $\rho_{b}$ since the Drude formula for conductivity in a quasi-1D metal is independent of the number of carriers [see, e.g., A. Narduzzo et al., New J. Phys. 8, 172 (2006)]. Extrapolating from high fields (Fig. 1), or from high temperatures [15], we obtain a lower bound of $\ell_{\text {tr }}$ within the chains of $\geq 1000 \AA$.

[25] T. J. B. M. Janssen et al., Phys. Rev. B 57, 11698 (1998).

[26] K. Yasui and T. Kita, Phys. Rev. B 66, 184516 (2002).

[27] P. Blaha et al., WIEN2K, An Augmented Plane Wave + Local Orbitals Program for Calculating Crystal Properties (Karlheinz Schwarz, Techn. Universität Wien, Austria, 2001), ISBN 3-9501031-1-2.

[28] P. Lightfoot et al., Acta Crystallogr. Sect. C 47, 1143 (1991).

[29] J. J. Yu, K. T. Park, and A. J. Freeman, Physica (Amsterdam) 172C, 467 (1991).

[30] C. Ambrosch-Draxl, P. Blaha, and K. Schwarz, Phys. Rev. B 44, 5141 (1991).

[31] Yelland et al. recently reported [, Phys. Rev. Lett. 100, 047003 (2008).] another study of quantum oscillations in Y124 using a high frequency susceptibility technique. Their data show oscillations with $\langle F\rangle=660 \pm 15 \mathrm{~T}$ and $m^{*}=3.0 \pm 0.3 m_{e}$, which are the same as the values reported here within the quoted error. 